\title{
ON SOME PROPERTIES OF CAUCHY PROBLEM FOR NON-STATIONARY THIRD ORDER COMPOSITE TYPE EQUATION
}

\section{A.R. KHASHIMOV, S. YAKUBOV}

\begin{abstract}
In the paper we construct a solution to the Cauchy problem for a non-stationary third order composite type equation and we study some of its properties.
\end{abstract}

Keywords: Cauchy problem, third order equations, non-stationary PDE, Airy functions, increasing at infinity solutions.

Mathematics Subject Classification: 35A02, 35A09, 35B40

\section{INTRODUCTION}

The aim of the present work is to study some properties of solution to equation

$$
\sum_{i=1}^{n} \frac{\partial^{3}}{\partial x_{i}^{3}}-\frac{\partial u}{\partial t}=0
$$

in the domain $D=\left\{\left(x_{i} ; t\right):-\infty<x_{i}<\infty, 0<t \leqslant T\right\}$ subject to the initial condition

$$
u\left(x_{1}, x_{2}, \ldots, x_{n}, 0\right)=\varphi\left(x_{1}, x_{2}, \ldots, x_{n}\right), \quad-\infty<x_{i}<\infty .
$$

If $n=1$ in (1), we obtain the equation

$$
u_{x x x}-u_{t}=0
$$

which was studied in work [2]. In this work, there were constructed the fundamental solution to equation (3) and potential theory, as well as there were developed the method of studying boundary value problems and Cauchy problem for equation (3). Later the solution to the Cauchy problem for equation (3) was constructed in work [16] in a wider class and some of its properties were studied. By the same method there was constructed a solution to the Cauchy problem for the equation of high odd order [15]

$$
\frac{\partial^{2 k+1} u}{\partial x^{2 k+1}}+(-1)^{k} \frac{\partial u}{\partial t}=0 .
$$

If we let $n=2$ in (1), we obtain the equation

$$
u_{x x x}+u_{y y y}-u_{t}=0 .
$$

We note that solutions to equation (4) and linear Zakharov-Kuznetsov equation (see [4], 5])

$$
u_{t}+u_{x x x}+u_{x y y}=0
$$

have similar aysmptotic properties at infinity. Zakharov-Kuznetsov equation (5) is one of possible generalizations to Korteweg-de-Vries equation in a multi-dimensional space and it

A.R. Khashimov, S. Yakubov, On some properties of Cauchy problem for non-Stationary THIRD ORDER COMPOSITE TYPE EQUATION.

(c) Khashimov A.R., Yakubov S. 2014.

The work is supported by GKNT RUz (grant F-4-55).

Submitted October 30, 2014. 
describes ion-acoustic wave processes in plasme [20]. The equation to the Cauchy problem for equation (4) was constructed in work [8].

The solvability class for the Cauchy problem in the classes of functions growing at infinity was determined first in work by A.N. Tikhonov [18] for the heat equation. Further study for differential equation of odd order in the classes of functions growing at infinity was made by means of the theory of generalized functions [9, 10, 11, 12, 13, 19]. At present, the most complete theory for linear equations of even order (for instance, for linear equations of parabolic type) was developed in [3, 14, 17].

In work [1] there was constructed the fundamental solution to equation (1) in space $\mathbb{R}^{n+1}$

$$
\begin{aligned}
& U\left(x_{1}-\xi_{1}, x_{2}-\xi_{2}, \ldots, x_{n}-\xi_{n} ; t-\tau\right) \\
&=\frac{1}{(t-\tau)^{\frac{n}{3}}} f\left(\frac{x_{1}-\xi_{1}}{(t-\tau)^{\frac{1}{3}}}\right) \ldots f\left(\frac{x_{n}-\xi_{n}}{(t-\tau)^{\frac{1}{3}}}\right), \quad x_{i} \neq \xi_{i}, \quad t>\tau, \quad i=\overline{1, n},
\end{aligned}
$$

where $f(z)=\int_{0}^{\infty} \cos \left(\lambda^{3}-\lambda z\right) d \lambda,-\infty<z<\infty$, is the Airy function satisfying the equation

$$
f(z)+\frac{1}{3} z f(z)=0
$$

Function $f(z)$ satisfies the following identities

$$
\int_{-\infty}^{\infty} f(z) d z=\pi, \quad \int_{-\infty}^{0} f(z) d z=\frac{\pi}{3}, \quad \int_{0}^{\infty} f(z) d z=\frac{2 \pi}{3}
$$

\section{MAIN RESULTS}

Theorem 1. Let $\varphi\left(x_{1}, \ldots, x_{n}\right)$ be a piece-wise continuous function with a compact support $D_{\left(a_{i}, b_{i}\right)}=\left\{x_{i}: a_{i} \leqslant x_{i} \leqslant b_{i}\right\}, i=\overline{1, n}, D_{\left(a_{i}, b_{i}\right)} \subset \mathbb{R}^{n}$ and having an bounded variation. Then the function

$$
u\left(x_{1}, \ldots, x_{n}, t\right)=\frac{1}{\pi^{n}} \int_{\mathbb{R}^{n}} U\left(x_{1}-\xi_{1}, \ldots, x_{n}-\xi_{n} ; t\right) \varphi\left(\xi_{1}, \ldots, \xi_{n}\right) d \xi_{1} \ldots d \xi_{n}
$$

satisfies equation (1) as $t>0$ and for each $x_{i}^{0} \in\left(a_{i}, b_{i}\right)$

$$
\lim _{t \rightarrow+0} u\left(x_{1}^{0}, \ldots, x_{n}^{0}, t\right)=\frac{2}{3} \varphi\left(x_{1}^{0}-0, \ldots, x_{n}^{0}-0\right)+\frac{1}{3} \varphi\left(x_{1}^{0}+0, \ldots, x_{n}^{0}+0\right) .
$$

The validity of the first part of the theorem follows immediately from the properties of the fundamental solution to equation (1) and the hypothesis of the theorem. The second part is proven separately w.r.t. each spatial variable. Since the proof of this part of the theorem is similar to work [16] and makes no essential troubles, we do not dwell on it.

Theorem 2. Let function $\varphi\left(x_{1}, \ldots, x_{n}\right)$ be continuous and have a bounded variation on each bounded domain $D_{\left(a_{i}, b_{i}\right)}=\left\{x_{i}: a_{i} \leqslant x_{i} \leqslant b_{i}\right\}, i=\overline{1, n}, D_{\left(a_{i}, b_{i}\right)} \subset \mathbb{R}^{n}$, and the variation of the function

$$
P(y)=y^{\frac{3}{4}+\delta_{1}} \psi(y)
$$

be bounded as $y<a_{0}$ for each $a_{0}=$ const. Moreover, let

$$
\varphi\left(x_{1}, \ldots, x_{n}\right) \sim \prod_{j} \psi\left(x_{j}\right) \exp \left\{\text { const } \sum_{i \neq j}\left|x_{i}\right|^{\frac{3}{2}-\delta_{2}}\right\}
$$


as $x_{i} \rightarrow \infty, x_{j}<a_{j}, j=\overline{1, n}, i+j=n$; and

$$
\varphi\left(x_{1}, \ldots, x_{n}\right) \sim \prod_{j} \psi\left(x_{j}\right)
$$

as $x_{j}<a_{j}$, where $\delta_{1}, \delta_{2}$ are positive numbers. Then the function

$$
u\left(x_{1}, \ldots, x_{n}, t\right)=\frac{1}{\pi^{n}} \int_{\mathbb{R}^{n}} U\left(x_{1}-\xi_{1}, \ldots, x_{n}-\xi_{n} ; t\right) \varphi\left(\xi_{1}, \ldots, \xi_{n}\right) d \xi_{1} \ldots d \xi_{n}
$$

satisfies equation (1) as $t>0$ and the condition

$$
\lim _{\left(x_{1}, \ldots, x_{n}, t\right) \rightarrow\left(x_{1}^{0}, \ldots, x_{n}^{0},+0\right)} u\left(x_{1}, \ldots, x_{n}, t\right)=\varphi\left(x_{1}^{0}, \ldots, x_{n}^{0}\right) .
$$

Proof. We begin by proving the first part of the theorem. We differentiate expression (10) w.r.t. $x_{j}$ to obtain

$$
\begin{aligned}
\pi^{n} \frac{\partial^{3} u}{\partial x_{j}^{3}} & =\int_{-\infty}^{\infty} \ldots \int_{-\infty}^{\infty} \frac{\partial^{3} U\left(x_{1}-\xi_{1}, \ldots, x_{n}-\xi_{n} ; t\right)}{\partial x_{j}^{3}} \varphi\left(\xi_{1}, \ldots, \xi_{n}\right) d \xi_{1} \ldots d \xi_{n} \\
& =\int_{-\infty}^{\infty} \ldots \int_{-\infty}^{\infty} \frac{\partial^{3} U\left(\xi_{1}, \ldots, \xi_{n} ; t\right)}{\partial x_{j}^{3}} \varphi\left(x_{1}-\xi_{1}, \ldots, x_{n}-\xi_{n}\right) d \xi_{1} \ldots d \xi_{n} .
\end{aligned}
$$

On the other hand,

$$
\frac{\partial^{3} U\left(x_{1}, \ldots, x_{n} ; t\right)}{\partial x_{j}^{3}}=-\frac{t^{-1}}{3}\left\{U+x_{j} U_{x_{j}}\right\} .
$$

While calculating the derivates we have made use of the identity (7). We get

$$
\begin{aligned}
\pi^{n} t \frac{\partial^{3} u}{\partial x_{j}^{3}}= & -\frac{1}{3} \int_{-\infty}^{\infty} \ldots \int_{-\infty}^{\infty} f\left(z_{1}\right) \ldots f\left(z_{n}\right) \varphi\left(\xi_{1}-z_{1} t^{\frac{1}{3}}, \ldots, \xi_{n}-z_{n} t^{\frac{1}{3}}\right) d z_{1} \ldots d z_{n} \\
& -\frac{1}{3} \int_{-\infty}^{\infty} \ldots \int_{-\infty}^{\infty} f\left(z_{1}\right) \ldots f\left(z_{j-1}\right) f\left(z_{j+1}\right) \ldots f\left(z_{n}\right) d z_{1} \ldots d z_{j-1} d z_{j+1} \ldots d z_{n} \\
& \cdot \int_{-\infty}^{\infty} z f^{\prime}\left(z_{j}\right) \varphi\left(\xi_{1}-z_{1} t^{\frac{1}{3}}, \ldots, \xi_{n}-z_{n} t^{\frac{1}{3}}\right) d z_{j}=-\frac{1}{3}\left\{u_{j 1}+u_{j 2}\right\}, z_{j}=\frac{x_{j}-\xi_{j}}{t^{\frac{1}{3}}} .
\end{aligned}
$$

Let us prove that under the hypothesis of the theorem the integral in the right hand side of (12) obtained by the formal differentiation converges. Let us study the convergence of integral (12) as $j=1$; other cases can be studied in the same way. Airy function satisfies the identities [16]:

$$
f(z) \sim\left\{\begin{array}{l}
|z|^{-\frac{1}{4}} \exp \left(-\frac{2}{3}|z|^{\frac{3}{2}}\right)\left(\sqrt{\pi}+O\left(|z|^{-\frac{3}{2}}\right)\right), \\
|z|^{\frac{1}{4}} \exp \left(-\frac{2}{3}|z|^{\frac{3}{2}}\right)\left(\sqrt{\pi}+O\left(|z|^{-\frac{3}{2}}\right)\right)
\end{array}\right.
$$

for sufficiently large negative $z$;

$$
f(z) \sim\left\{\begin{array}{l}
z^{-\frac{1}{4}} \cos \left(\frac{2}{3}|z|^{\frac{3}{2}}-\frac{\pi}{4}\right)\left(\sqrt{\pi}+O\left(|z|^{-\frac{3}{2}}\right)\right), \\
z^{\frac{1}{4}} \sin \left(\frac{2}{3}|z|^{\frac{3}{2}}-\frac{\pi}{4}\right)\left(\sqrt{\pi}+O\left(|z|^{-\frac{3}{2}}\right)\right),
\end{array}\right.
$$


for sufficiently large positive $z$.

Let $j=1, t \geqslant t_{0}>0, a_{i} \leqslant x_{i} \leqslant b_{i}, i=2,3, \ldots, n$.

We first consider the second term in the right hand side of (12). Then by (12) we have

$$
\begin{aligned}
u_{12}\left(x_{1}, x_{2}, \ldots, x_{n}, t\right)=\left\{\int_{-\infty}^{-r_{2}}+\int_{-r_{2}}^{r_{2}}+\int_{r_{2}}^{\infty}\right\} f\left(z_{2}\right) d z_{2} \ldots\left\{\int_{-\infty}^{-r_{n}}+\int_{-r_{n}}^{r_{n}}+\int_{r_{n}}^{\infty}\right\} f\left(z_{n}\right) d z_{n} \\
\cdot\left\{\int_{-\infty}^{-r_{1}}+\int_{-r_{1}}^{r_{1}}+\int_{r_{1}}^{\infty}\right\} z_{1} f^{\prime}\left(z_{1}\right) \varphi\left(x_{1}-z_{1} t^{\frac{1}{3}}, \ldots, x_{n}-z_{n} t^{\frac{1}{3}}\right) d z_{1} \\
=\left\{\int_{-\infty}^{-r_{2}}+\int_{-r_{2}}^{r_{2}}+\int_{r_{2}}^{\infty}\right\} f\left(z_{2}\right) d z_{2} \ldots\left\{\int_{-\infty}^{-r_{n}}+\int_{-r_{n}}^{r_{n}}+\int_{r_{n}}^{\infty}\right\}\left[J_{1}+J_{2}+J_{3}\right] f\left(z_{n}\right) d z_{n},
\end{aligned}
$$

where $r_{j}$ are sufficiently large positive numbers. First we consider the integrals involving the expression $J_{1}\left(x_{1}, \ldots, x_{n} ; z_{2}, \ldots, z_{n} ; t\right)$ for sufficiently large positive $r_{1}$.

Let $z_{\in}\left[-\infty ;-r_{i}, \quad i=\overline{2, n}\right]$. Then by condition $(13)$ we have

$$
\begin{aligned}
\int_{-\infty}^{-r_{2}} f & \left(z_{2}\right) d z_{2} \ldots \int_{-\infty}^{-r_{n}} J_{1}\left(x_{1}, \ldots, x_{n} ; z_{2}, \ldots, z_{n} ; t\right) f\left(z_{n}\right) d z_{n} \\
& =\int_{-\infty}^{-r_{2}} f\left(z_{2}\right) d z_{2} \ldots \int_{-\infty}^{-r_{n}} f\left(z_{n}\right) d z_{n} \int_{-\infty}^{-r_{1}} z_{1} f^{\prime}\left(z_{1}\right) \varphi\left(x_{1}-z_{1} t^{\frac{1}{3}}, \ldots, x_{n}-z_{n} t^{\frac{1}{3}}\right) d z_{1} \\
& O\left(\int_{r_{2}}^{\infty} z_{2}^{-\frac{1}{4}} \exp \left[-z_{2}^{\frac{3}{2}}\left(\frac{2}{3}-C z_{2}^{-\delta_{2}}\left(\frac{x_{2}}{z_{2}}+t^{\frac{1}{3}}\right)^{\frac{3}{2}-\delta_{2}}\right)\right] d z_{2}\right) \cdot \ldots \\
\cdot & O\left(\int_{r_{n}}^{\infty} z_{n}^{-\frac{1}{4}} \exp \left[-z_{n}^{\frac{3}{2}}\left(\frac{2}{3}-C z_{n}^{-\delta_{2}}\left(\frac{x_{n}}{z_{n}}+t^{\frac{1}{3}}\right)^{\frac{3}{2}-\delta_{2}}\right)\right] d z_{n}\right) \\
\cdot & O\left(\int_{r_{1}}^{\infty} z_{1}^{-\frac{1}{4}} \exp \left[-z_{1}^{\frac{3}{2}}\left(\frac{2}{3}-C z_{1}^{-\delta_{2}}\left(\frac{x_{1}}{z_{1}}+t^{\frac{1}{3}}\right)^{\frac{3}{2}-\delta_{2}}\right)\right] d z_{1}\right) .
\end{aligned}
$$

We see that this integral converges uniformly to zero as $r_{j} \rightarrow \infty$.

Suppose now that $z_{2} \in\left[r_{2}, \infty\right], z_{k} \in\left[-\infty,-r_{k}\right], k=\overline{3, n}$. Then by 13 and the hypothesis of the theorem we have

$$
\begin{aligned}
& \int_{r_{2}}^{\infty} f\left(z_{2}\right) d z_{2} \int_{-\infty}^{-r_{3}} f\left(z_{3}\right) d z_{3} \ldots \int_{-\infty}^{-r_{n}} J_{1}\left(x_{1}, \ldots, x_{n} ; z_{2}, \ldots, z_{n} ; t\right) f\left(z_{n}\right) d z_{n}= \\
& \quad=\int_{r_{2}}^{\infty} f\left(z_{2}\right) d z_{2} \int_{-\infty}^{-r_{3}} f\left(z_{3}\right) d z_{3} \ldots \int_{-\infty}^{-r_{n}} f\left(z_{n}\right) d z_{n} \int_{-\infty}^{-r_{1}} z_{1} f^{\prime}\left(z_{1}\right) \varphi\left(x_{1}-z_{1} t^{\frac{1}{3}}, \ldots, x_{n}-z_{n} t^{\frac{1}{3}}\right) d z_{1} \\
& \quad \sim O\left(\int_{r_{1}}^{\infty} z_{1}^{\frac{5}{4}} \exp \left[-z_{1}^{\frac{3}{2}}\left(\frac{2}{3}-C z_{1}^{-\delta_{2}}\left(\frac{x_{1}}{z_{1}}+t^{\frac{1}{3}}\right)^{\frac{3}{2}-\delta_{2}}\right)\right] d z_{1}\right) \ldots
\end{aligned}
$$




$$
\begin{aligned}
& \cdot O\left(\int_{r_{n}}^{\infty} z_{n}^{-\frac{1}{4}} \exp \left[-z_{n}^{\frac{3}{2}}\left(\frac{2}{3}-C z_{n}^{-\delta_{2}}\left(\frac{x_{n}}{z_{n}}+t^{\frac{1}{3}}\right)^{\frac{3}{2}-\delta_{2}}\right)\right] d z_{n}\right) \int_{r_{2}}^{\infty} \psi\left(x_{2}-z_{2} t^{\frac{1}{3}}\right) f\left(z_{2}\right) d z_{2} \\
& =J_{11}\left(x_{1}, t\right) \ldots J_{1 n}\left(x_{n}, t\right) J_{12}\left(x_{2}, t\right) .
\end{aligned}
$$

The convergence of integrals $J_{11}\left(x_{1}, t\right), \ldots, J_{1 n}\left(x_{n}, t\right)$ to zero as $r_{j} \rightarrow \infty, j=1,3, \ldots, n$, is obvious. We consider integral $J_{12}\left(x_{2}, t\right)$ :

$$
\begin{aligned}
\int_{r_{2}}^{\infty} \psi\left(x_{2}-z_{2} t^{\frac{1}{3}}\right) f\left(z_{2}\right) d z_{2} & \sim \int_{r_{2}}^{\infty} z_{2}^{-\frac{1}{4}} \cos \left(\frac{2}{3} z_{2}^{\frac{3}{2}}-\frac{\pi}{4}\right) \psi\left(x_{2}-z_{2} t^{\frac{1}{3}}\right) d z_{2} \\
& +O\left(\int_{r_{2}}^{\infty} z_{2}^{-\frac{7}{4}} \cos \left(\frac{2}{3} z_{2}^{\frac{3}{2}}-\frac{\pi}{4}\right) \psi\left(x_{2}-z_{2} t^{\frac{1}{3}}\right) d z_{2}\right) .
\end{aligned}
$$

Here

$$
\begin{aligned}
& \left|\int_{r_{2}}^{\infty} z_{2}^{-\frac{1}{4}} \cos \left(\frac{2}{3} z_{2}^{\frac{3}{2}}-\frac{\pi}{4}\right) \psi\left(x_{2}-z_{2} t^{\frac{1}{3}}\right) d z_{2}\right| \\
& =\left|\int_{r_{2}}^{\infty} z_{2}^{-1-\delta_{1}} \cos \left(\frac{2}{3} z_{2}^{\frac{3}{2}}-\frac{\pi}{4}\right)\right| \frac{x_{2}}{z_{2}}-\left.t^{\frac{1}{3}}\right|^{-\frac{3}{4}-\delta_{1}}\left|x_{2}-z_{2} t^{\frac{1}{3}}\right|^{\frac{3}{4}+\delta_{1}} \psi\left(x_{2}-z_{2} t^{\frac{1}{3}}\right) d z_{2} \mid \\
& \leqslant M \int_{r_{2}}^{\infty} z_{2}^{-1-\delta_{1}} d z_{2}=\left(\frac{M}{\delta_{1}}\right) r_{2}^{-\delta_{1}}, \\
& \left|\int_{r_{2}}^{\infty} z_{2}^{-\frac{7}{4}} \cos \left(\frac{2}{3} z_{2}^{\frac{3}{2}}-\frac{\pi}{4}\right) \psi\left(x_{2}-z_{2} t^{\frac{1}{3}}\right) d z_{2}\right| \\
& =\left|\int_{r_{2}}^{\infty} z_{2}^{-\frac{5}{2}-\delta_{1}} \cos \left(\frac{2}{3} z_{2}^{\frac{3}{2}}-\frac{\pi}{4}\right)\right| \frac{x_{2}}{z_{2}}-\left.t^{\frac{1}{3}}\right|^{-\frac{3}{4}-\delta_{1}}\left|x_{2}-z_{2} t^{\frac{1}{3}}\right|^{\frac{3}{4}+\delta_{1}} \psi\left(x_{2}-z_{2} t^{\frac{1}{3}}\right) d z_{2} \mid \\
& \leqslant M \int_{r_{2}}^{\infty} z_{2}^{-\frac{5}{2}-\delta_{1}} d z_{2}=\left(\frac{M}{\delta_{1}}\right) r_{2}^{-\frac{3}{2}-\delta_{1}} .
\end{aligned}
$$

This is why integral $J_{12}\left(x_{2}, t\right)$ converges to zero as $r_{2} \rightarrow \infty$. In the same we prove the convergence of the other integrals involving the expression $J_{1}\left(x_{1}, \ldots, x_{n}, z_{2}, \ldots, z_{n}, t\right)$.

In what follows we shall make use of the following theorem.

Theorem 3 ([7]). Suppose that the variation of a function $P(x)$ is bounded on an interval $(a, b)$ and

$$
\left|\int_{a}^{b} Q(x) d x\right|<M
$$

Then

$$
\left|\int_{a}^{b} Q(x) P(x) d x\right|<M\left\{|P(x)|+V_{a}^{b}(P(x))\right\},
$$

where $V_{a}^{b}$ is the variation of a function on interval $(a, b)$. 
We proceed to the integrals involving the expression $J_{3}\left(x_{1}, \ldots, x_{n}, z_{2}, \ldots, z_{n}, t\right)$ for sufficiently large positive $r_{1}$.

Let $z_{i}^{\prime} \in\left[-\infty,-r_{i}^{\prime}\right]$. Then by (13) the hypothesis of the theorem we have

$$
\begin{aligned}
\int_{-\infty}^{-r_{2}} f & \left(z_{2}\right) d z_{2} \ldots \int_{-\infty}^{-r_{n}} J_{3}\left(x_{1}, \ldots, x_{n}, z_{2}, \ldots, z_{n}, t\right) d z_{n} \\
& =\int_{-\infty}^{-r_{2}} f\left(z_{2}\right) d z_{2} \ldots \int_{-\infty}^{-r_{n}} f\left(z_{n}\right) d z_{n} \int_{r_{1}}^{\infty} z_{1} f^{\prime}\left(z_{1}\right) \varphi\left(x_{1}-z_{1} t^{\frac{1}{3}}, \ldots, x_{n}-z_{n} t^{\frac{1}{3}}\right) d z_{1} \\
& \sim\left(\int_{r_{2}}^{\infty} z_{2}^{-\frac{1}{4}} \exp \left[-z_{2}^{\frac{3}{2}}\left(\frac{2}{3}-C z_{2}^{-\delta_{2}}\left(\frac{x_{2}}{z_{2}}+t^{\frac{1}{3}}\right)^{\frac{3}{2}-\delta_{2}}\right)\right] d z_{2}\right) \cdot \ldots \\
\cdot & O\left(\int_{r_{n}}^{\infty} z_{n}^{-\frac{1}{4}} \exp \left[-z_{n}^{\frac{3}{2}}\left(\frac{2}{3}-C z_{n}^{-\delta_{2}}\left(\frac{x_{n}}{z_{n}}+t^{\frac{1}{3}}\right)^{\frac{3}{2}-\delta_{2}}\right)\right] d z_{n}\right) \\
& \cdot \int_{r_{1}}^{\infty} \psi\left(x_{1}-z_{1} t^{\frac{1}{3}}\right) z_{1} f^{\prime}\left(z_{1}\right) d z_{1}=J_{32}\left(x_{2}, t\right) \ldots J_{3 n}\left(x_{n}, t\right) J_{31}\left(x_{1}, t\right) .
\end{aligned}
$$

It is obvious that as $r_{i}^{\prime} \rightarrow \infty$, integral $J_{32}\left(x_{2}, t\right), \ldots, J_{3 n}\left(x_{n}, t\right)$ converges to zero. We consider integral $J_{31}\left(x_{1}, t\right)$ :

$$
\begin{aligned}
& \int_{r_{1}}^{\infty} \psi\left(x_{1}-z_{1} t^{\frac{1}{3}}\right) z_{1} f^{\prime}\left(z_{1}\right) d z_{1} \\
& \sim \int_{r_{1}}^{\infty} z_{1}^{\frac{5}{4}} \sin \left(\frac{2}{3} z_{1}^{\frac{3}{2}}-\frac{\pi}{4}\right) \psi\left(x_{1}-z_{1} t^{\frac{1}{3}}\right) d z_{1}+O\left(\int_{r_{1}}^{\infty} z_{1}^{-\frac{1}{4}} \sin \left(\frac{2}{3} z_{2}^{\frac{3}{2}}-\frac{\pi}{4}\right) \psi\left(x_{1}-z_{1} t^{\frac{1}{3}}\right) d z_{1}\right) .
\end{aligned}
$$

It is clear that as $r_{1} \rightarrow \infty$, the second integral in the right hand side of this relation converges to zero. This is why it is sufficient to study just the first integral in the right hand side:

$$
\begin{aligned}
& \int_{r_{1}}^{\infty} z_{1}^{\frac{5}{4}} \sin \left(\frac{2}{3} z_{1}^{\frac{3}{2}}-\frac{\pi}{4}\right) \psi\left(x_{1}-z_{1} t^{\frac{1}{3}}\right) d z_{1} \\
& =\int_{r_{1}}^{\infty} z_{1}^{\frac{1}{2}-\delta_{1}} \sin \left(\frac{2}{3} z_{1}^{\frac{3}{2}}-\frac{\pi}{4}\right)\left(\frac{x_{1}}{z_{1}}-t^{\frac{1}{3}}\right)^{-\frac{3}{4}-\delta_{1}}\left(x_{1}-z_{1} t^{\frac{1}{3}}\right)^{\frac{3}{4}+\delta_{1}} \psi\left(x_{1}-z_{1} t^{\frac{1}{3}}\right) d z_{1} \\
& =\frac{2}{3} \int_{\rho}^{\infty} v^{-\frac{2}{3} \delta_{1}} \sin \left(\frac{2}{3} v-\frac{\pi}{4}\right) \mu(v)\left\{\left(x_{1}-v^{\frac{2}{3}} t^{\frac{1}{3}}\right)^{\frac{3}{4}+\delta_{1}} \psi\left(x_{1}-v^{\frac{2}{3}} t^{\frac{1}{3}}\right)\right\} d v
\end{aligned}
$$

where $\rho=r_{1}^{\frac{3}{2}}, \mu(v)=\left(\frac{x_{1}}{v^{\frac{2}{3}}}-t^{\frac{1}{3}}\right)^{-\frac{3}{4}-\delta_{1}}$. For sufficiently large positive $r_{1}$, the absolute value of this integral is bounded by

$$
\frac{2}{3}\left\{\left|x_{1}-r_{1} t^{\frac{1}{3}}\right|^{\frac{3}{4}+\delta_{1}}\left|\psi\left(x_{1}-r_{1} t^{\frac{1}{3}}\right)\right|+V\left[\left(x_{1}-v^{\frac{2}{3}} t^{\frac{1}{3}}\right)^{\frac{3}{4}+\delta_{1}} \psi\left(x_{1}-v^{\frac{2}{3}} t^{\frac{1}{3}}\right) ; v \geqslant r_{1}^{\frac{3}{2}}\right]\right\}
$$




$$
\cdot \sup \left\{\left|\int_{m}^{n} v^{-\frac{2}{3} \delta_{1}} \sin \left(\frac{2}{3} v-\frac{\pi}{4}\right) d v\right|\right\}
$$

where $r_{1}^{\frac{3}{2}} \leqslant m<n$.

The existence of the integrals (see [6])

$$
\begin{aligned}
& \int_{0}^{\infty} x^{p} \sin (a x+b) d x=a^{\frac{1}{p+1}} \Gamma(1+p) \cos \left(b+\frac{p \pi}{2}\right), \quad a>0, \quad-1<p<0, \\
& \int_{0}^{\infty} x^{p} \cos (a x+b) d x=-a^{\frac{1}{p+1}} \Gamma(1+p) \sin \left(b+\frac{p \pi}{2}\right), \quad a<0, \quad-1<p<0
\end{aligned}
$$

means that the expression under the sup converges to zero as $r_{1} \rightarrow \infty$. Therefore, integral $J_{31}\left(x_{1}, t\right)$ converges to zero uniformly as $r_{1} \rightarrow \infty$.

Suppose that $z_{2} \in\left[r_{2}, \infty\right), z_{k} \in\left(-\infty,-r_{k}\right], k=\overline{3, n}$. Then by $(13)$ and the hypothesis of the theorem we have

$$
\begin{aligned}
\int_{r_{2}}^{\infty} f\left(z_{2}\right) d z_{2} & \int_{-\infty}^{-r_{3}} f\left(z_{3}\right) d z_{3} \ldots \int_{-\infty}^{-r_{n}} J_{3}\left(x_{1}, \ldots, x_{n}, z_{2}, \ldots, z_{n}, t\right) d z_{n} \\
= & \int_{r_{2}}^{\infty} f\left(z_{2}\right) d z_{2} \ldots \int_{-\infty}^{-r_{n}} f\left(z_{n}\right) d z_{n} \int_{r_{1}}^{\infty} z_{1} f^{\prime}\left(z_{1}\right) \varphi\left(x_{1}-z_{1} t^{\frac{1}{3}}, \ldots, x_{n}-z_{n} t^{\frac{1}{3}}\right) d z_{1} \\
\sim & O\left(\int_{r_{3}}^{\infty} z_{3}^{-\frac{1}{4}} \exp \left[-z_{3}^{\frac{3}{2}}\left(\frac{2}{3}-C z_{3}^{-\delta_{2}}\left(\frac{x_{3}}{z_{3}}+t^{\frac{1}{3}}\right)^{\frac{3}{2}-\delta_{2}}\right)\right] d z_{3}\right) \ldots \\
& \cdot O\left(\int_{r_{n}}^{\infty} z_{n}^{-\frac{1}{4}} \exp \left[-z_{n}^{\frac{3}{2}}\left(\frac{2}{3}-C z_{n}^{-\delta_{2}}\left(\frac{x_{n}}{z_{n}}+t^{\frac{1}{3}}\right)^{\frac{3}{2}-\delta_{2}}\right)\right] d z_{n}\right) \\
& \cdot \int_{r_{2}}^{\infty} \psi\left(x_{2}-z_{2} t^{\frac{1}{3}}\right) f\left(z_{2}\right) d z_{2} \int_{r_{1}}^{\infty} \psi\left(x_{1}-z_{1} t^{\frac{1}{3}}\right) z_{1} f^{\prime}\left(z_{1}\right) d z_{1} \\
= & J_{33}\left(x_{2}, t\right) \ldots J_{3 n}\left(x_{n}, t\right) J_{32}\left(x_{2}, t\right) J_{31}\left(x_{1}, t\right) .
\end{aligned}
$$

The convergence of integrals $J_{33}\left(x_{2}, t\right), \ldots, J_{3 n}\left(x_{n}, t\right), J_{32}\left(x_{2}, t\right), J_{31}\left(x_{1}, t\right)$ follows from (16) and Theorem 3.

In the same we prove the convergence of the integrals involving the expression $J_{3}\left(x_{1}, \ldots, x_{n}, z_{1}, \ldots, z_{n}, t\right)$.

Thus, we have proven that integral (12) converges uniformly in $D_{\left(a_{i}, b_{i}\right)}$. Therefore, by the arbitrariness of $a_{i}, b_{i}$, and $t_{0}$ integral (12) converges uniformly in $D$.

Let us prove identity (11). We consider functions $\bar{\varphi}\left(x_{1}, \ldots, x_{n}\right)$ with a compact support. We assume that $a_{i}+1 \leqslant x_{i}^{0} \leqslant b_{i}-1$. We let $\bar{\varphi}\left(x_{1}, \ldots, x_{n}\right)=\Phi\left(a_{i}, b_{i}\right) \varphi\left(x_{1}, \ldots, x_{n}\right)$, where

$$
\Phi\left(a_{i}, b_{i}\right)=\left\{\begin{array}{lll}
1, & \text { if } & x_{i} \in D_{\left(a_{i}, b_{i}\right)} \\
0, & \text { if } & x_{i} \notin D_{\left(a_{i}, b_{i}\right)}
\end{array}\right.
$$

Let

$$
\bar{u}\left(x_{1}, \ldots, x_{n}, t\right)=\frac{1}{\pi^{n}} \int_{\mathbb{R}^{n}} U\left(x_{1}-\xi_{1}, \ldots, x_{n}-\xi_{n} ; t\right) \bar{\varphi}\left(\xi_{1}, \ldots, \xi_{n}\right) d \xi_{1} \ldots d \xi_{n}
$$


We consider the difference

$$
v\left(x_{1}, \ldots, x_{n}, t\right)=u\left(x_{1}, \ldots, x_{n}, t\right)-\bar{u}\left(x_{1}, \ldots, x_{n}, t\right), a_{i}+1 \leqslant x_{i} \leqslant b_{i}-1 .
$$

We have

$$
\begin{aligned}
v\left(x_{1}, \ldots, x_{n}, t\right)= & \int_{-\infty}^{-k_{1}} f\left(z_{1}\right) d z_{1} \ldots \int_{-\infty}^{-k_{n}} f\left(z_{n}\right) \varphi\left(x_{1}-z_{1} t^{\frac{1}{3}}, \ldots, x_{n}-z_{n} t^{\frac{1}{3}}\right) d z_{n} \\
& +\int_{h_{1}}^{\infty} f\left(z_{1}\right) d z_{1} \ldots \int_{h_{n}}^{\infty} f\left(z_{n}\right) \varphi\left(x_{1}-z_{1} t^{\frac{1}{3}}, \ldots, x_{n}-z_{n} t^{\frac{1}{3}}\right) d z_{n} \\
= & v_{1}\left(x_{1}, \ldots, x_{n}, t\right)+v_{2}\left(x_{1}, \ldots, x_{n}, t\right)
\end{aligned}
$$

where $k_{i}=\left(b_{i}-x_{i}\right) t^{-\frac{1}{3}}, h_{i}=\left(x_{i}-a_{i}\right) t^{-\frac{1}{3}}$.

By identities (13) and the hypothesis of the theorem for sufficiently large $k_{i}$ we obtain

$$
\begin{aligned}
v_{1}\left(x_{1}, \ldots, x_{n}, t\right)= & O\left(\int_{k_{1}}^{\infty} z_{1}^{-\frac{1}{4}} \exp \left(-\frac{2}{3} z_{1}^{\frac{3}{2}}+C\left(x_{1}-z_{1} t^{\frac{1}{3}}\right)^{\frac{3}{2}-\delta_{2}}\right) d z_{1}\right) \cdot \ldots \\
& \cdot O\left(\int_{k_{1}}^{\infty} z_{n}^{-\frac{1}{4}} \exp \left(-\frac{2}{3} z_{n}^{\frac{3}{2}}+C\left(x_{n}-z_{n} t^{\frac{1}{3}}\right)^{\frac{3}{2}-\delta_{2}}\right) d z_{n}\right) .
\end{aligned}
$$

Hence, $v_{1}\left(x_{1}, \ldots, x_{n}, t\right)$ tends to zero as $t \rightarrow+0, k_{i} \rightarrow \infty$.

By Theorem 3, the second integral in (16) can be estimated as

$$
\begin{aligned}
\left|v_{2}\left(x_{1}, \ldots, x_{n}, t\right)\right| \leqslant & \left\{\left|\varphi\left(x_{1}-h_{1} t^{\frac{1}{3}} \ldots x_{n}-h_{n} t^{\frac{1}{3}}\right)\right|\right. \\
& \left.+V\left(\varphi\left(x_{1}-z_{1} t^{\frac{1}{3}} \ldots x_{n}-z_{n} t^{\frac{1}{3}}\right) ; z_{i} \geqslant h_{i}\right)\right\} A\left(\alpha_{i}, \beta_{i}\right),
\end{aligned}
$$

where

$$
A\left(\alpha_{i}, \beta_{i}\right)=\sup \left\{\left|\int_{\alpha_{1}}^{\beta_{1}} f\left(z_{1}\right) d z_{1} \ldots \int_{\alpha_{n}}^{\beta_{n}} f\left(z_{n}\right) d z_{n}\right|: h_{i} \leqslant \alpha_{i} \leqslant \beta_{i}\right\} .
$$

Under the hypothesis of the theorem the first factor is bounded. Let us study $A\left(\alpha_{i}, \beta_{i}\right)$ for sufficiently large $h_{i} \leqslant \alpha_{i} \leqslant \beta_{i}$. We have

$$
\int_{\alpha_{1}}^{\beta_{1}} f\left(z_{1}\right) d z_{1} \ldots \int_{\alpha_{n}}^{\beta_{n}} f\left(z_{n}\right) d z_{n}
$$

Let us estimate the first integral; other integrals can be estimates in the same way:

$$
\begin{aligned}
\int_{\alpha_{1}}^{\beta_{1}} f\left(z_{1}\right) d z_{1} & \sim \int_{\alpha_{1}}^{\beta_{1}} z_{1}^{-\frac{1}{4}} \cos \left(\frac{2}{3} z_{1}^{\frac{3}{2}}-\frac{\pi}{4}\right)\left(\sqrt{\pi}+O\left(z_{1}^{-\frac{3}{2}}\right)\right) d z_{1} \sim \\
& \sim \int_{\gamma}^{\tau} \nu^{-\frac{1}{2}} \cos \left(\frac{2}{3} \nu-\frac{\pi}{4}\right)\left(\sqrt{\pi}+O\left(\nu^{-1}\right)\right) d \nu,
\end{aligned}
$$

where $\gamma=\alpha_{1}^{\frac{3}{2}}, \tau=\beta_{1}^{\frac{3}{2}}$. 
The first term in 17 can be estimated as follows:

$$
\begin{aligned}
\left|\int_{\gamma}^{\tau} \nu^{-\frac{1}{2}} \cos \left(\frac{2}{3} \nu-\frac{\pi}{4}\right) d \nu\right| \leqslant & C\left|\nu^{-\frac{1}{2}} \sin \left(\frac{2}{3} \nu-\frac{\pi}{4}\right)\right|_{\nu=\gamma}^{\nu=\tau} \\
& +C\left|\int_{\gamma}^{\tau} \nu^{-\frac{3}{2}} \sin \left(\frac{2}{3} \nu-\frac{\pi}{4}\right)\right| \leqslant \gamma^{-\frac{1}{2}}+\tau^{-\frac{1}{2}}+C\left(\gamma^{-\frac{1}{2}}-\tau^{-\frac{1}{2}}\right) .
\end{aligned}
$$

The second term is estimated by the expression $2\left(\gamma^{-\frac{1}{2}}-\tau^{-\frac{1}{2}}\right)$. We finally have

$$
\sup \left|\int_{\alpha_{1}}^{\beta_{1}} f\left(z_{1}\right) d z_{1}\right| \leqslant C\left(\beta_{1}^{-\frac{3}{4}}+\alpha_{1}^{-\frac{3}{4}}\right) \text {. }
$$

Thus, as $t \rightarrow+0, h_{i} \rightarrow \infty$, integral (17) uniformly converges to zero.

By means of the above theorem we can study the character of the growth for solutions $u\left(x_{1}, \ldots, x_{n}, t\right)$ to the problem. For the sake of simplicity we study the such growth w.r.t. variable $x_{1}$.

By (10), for sufficiently large positive numbers $r_{1}$ we get

$$
\begin{aligned}
u( & \left.x_{1}, \ldots, x_{n}, t\right)=\frac{1}{\pi^{n}} \int_{\mathbb{R}^{n}} U\left(x_{1}-\xi_{1}, \ldots, x_{n}-\xi_{n} ; t\right) \varphi\left(\xi_{1}, \ldots, \xi_{n}\right) d \xi_{1} \ldots d \xi_{n} \\
= & \frac{1}{\pi^{n}} \int_{\mathbb{R}^{n}} U\left(\xi_{1}, \ldots, \xi_{n} ; t\right) \varphi\left(\xi_{1}-\xi_{1}, \ldots, \xi_{n}-\xi_{n}\right) d \xi_{1} \ldots d \xi_{n} \\
= & \int_{-\infty}^{\infty} f\left(z_{2}\right) d z_{2} \ldots \int_{-\infty}^{\infty} f\left(z_{n}\right) d z_{n}\left\{\int_{-\infty}^{-r_{1}}+\int_{-r_{1}}^{r_{1}}+\int_{r_{1}}^{\infty}\right\} f\left(z_{1}\right) \varphi\left(x_{1}-z_{1} t^{\frac{1}{3}}, \ldots, x_{n}-z_{n} t^{\frac{1}{3}}\right) d z_{1} \\
= & u_{1}\left(x_{1}, \ldots, x_{n}, t\right)+u_{2}\left(x_{1}, \ldots, x_{n}, t\right)+u_{3}\left(x_{1}, \ldots, x_{n}, t\right) .
\end{aligned}
$$

In view of 13), 14 and the hypothesis of Theorem 2 we have

$$
\begin{aligned}
& \left|u_{1}\left(x_{1}, \ldots, x_{n}, t\right)\right| \leqslant K \exp \left\{\left|x_{1}\right|^{\frac{3}{2}-\delta_{2}}\right\}, \\
& \left|u_{3}\left(x_{1}, \ldots, x_{n}, t\right)\right| \leqslant M\left|x_{1}\right|^{-\frac{3}{4}-\delta_{1}} .
\end{aligned}
$$

Since $u_{2}\left(x_{1}, \ldots, x_{n}, t\right)$ is a bounded function, estimates $(18),(19)$ follow that the solution to the Cauchy problem can grows exponentially at the infinity the growth rate does not exceed $\exp \left\{\left|x_{1}\right|^{\frac{3}{2}-\delta_{2}}\right\}$, where $\delta_{2}>0$.

\section{BIBLIOGRAPHY}

1. S. Abdinazarov, Z.A. Sobirov. On fundamental solutions to equation with multiple characteristics of third order in a multi-dimensional space // Proc. International Scientific Conference "Partial differential equations and related problems of analysis and informatics", Tashkent. 12-13 (2004). (in Russian.)

2. L. Cattabriga. Potenzial di linea e di domino per equazione non parabolica in due variable a characteristiche multiple// Rend. Sem. Mat. Univ. Padova. 31, 1-45 (1961). (in Italian).

3. A. Friedman. Partial differential equations of parabolic type. Prentice-Hall, New Jersey (1964).

4. A.V. Famiskii and E.S. Baykova. On initial-boundary value problems in a strip for generalized two-dimensional Zakharov-Kuznetsov equation // Adv. Diff. Equat. 18:7/8, 663-686 (2013). 
5. A.V. Faminskii Well-posed initial-boundary value prolems for the Zakharov-Kuznetsov equation // Elect. J. Diff. Equat. 2008:127, 1-23 (2008).

6. I.S. Gradshteyn, I.M. Ryzhik. Table of integrals, series, and products. Nauka, Moscow (1971). [Academic Press, New York (1980).]

7. E.W. Hobson. Theory of functions of real variable. V. 1. Cambridge Univ. Press, Cambridge (1927).

8. A.R. Khashimov, Zh.Sh. Matnazarov. Cauchy problem for for non-stationary third order composite type equation // Uzb. Matem. Zhurn. 3, 9-10 (2009). (in Russian).

9. I. Kopachek, O.A. Oleinik. On asymptotic properties of solutions of the system of equations of elasticity theory // Uspekhi Matem. Nauk. 33:5, 189-190 (1978). [Russ. Math. Surv. 33:5, 197198 (1978).]

10. O.A. Oleinik, G.A. Iosif'yan. A priori estimates of the solutions of the first boundary value problem for the system of equations of elasticity theory, and their applications // Uspekhi Matem. Nauk. 32:5(197), 193-194 (1977). (in Russian).

11. O.A. Oleinik. The behavior of the solutions of linear parabolic systems of differential equations in unbounded domains // Uspekhi Matem. Nauk. 30:2(182), 219-220 (1975). (in Russian).

12. O.A. Oleinik, G.A. Yosifian. On singularities at the boundary points and uniqueness theorems of the first boundary value problem of elasticity // Comm. Partial Diff. Equations. 2: 9, 937-969 (1977).

13. O.A. Oleinik, G.A. Iosif'yan and I.N. Tarkhelidze. Bounds for the solutions of a biharmonic equation in the neighbourhood of non-regular boundary points and at infinity // Uspekhi Matem. Nauk. 33:3, 181-182 (1978). [Russ. Math. Surv. 33:3, 169-170 (1978).]

14. A.M. Il'in, A.S. Kalashnikov and O.A. Oleinik. Linear equations of the second order of parabolic type // Uspekhi Matem. Nauk. 17:3(105), 3-141 (1962). [Russ. Math. Surv. 17:3, 1-143 (1962).]

15. O.T. Kurbonov. On solvability of Cauchy problem for odd order equation with multiple characteristics // Uzb. Matem. Zhurn. 3, 33-38 (1998). (in Russian).

16. E.L. Roetman. Some observations about an odd order parabolic equation // J. Diff. Equat. 9:2, 335-345 (1971).

17. V.A. Solonnikov. On boundary value problems for linear parabolic systems of differential equations of general form // Trudy Mat. Inst. Steklov. 83, 3-163 (1965). [Proc. Steklov Inst. Math. 83, $1-184(1965)$.]

18. A. Tychonoff. Théorèmes d'unicité pour l'équation de la chaleur // Matem. Sbornik. 42:2, 199216 (1935). (in French).

19. N. Weck. An explicity Saint Venant's principle in tree-demensional elasticity // Proc. of 4th Conf. "Ordinary and Partial Differential Equations". Lect. Notes Math. 564, 518-526 (1976).

20. V.E. Zakharov and E.A. Kuznetsov. Three-dimentional solitons // Zhurn. Eksp. Teoret. Fiz. 66:2, 594-597 (1974). [Soviet Phys. JETP. 39:2, 285-288 (1974).]

Abdukomil Risbekovich Khashimov,

Tashkent Finance Institute,

A. Temur str., 60A,

100000, Tashkent, Uzbekistan

E-mail: abdukomil@yandex.ru

Sobitkhon Yakubov,

Tashkent Finance Institute,

A. Temur str., 60A,

100000, Tashkent, Uzbekistan

E-mail: abdukomil@yandex.ru 